\title{
Influence of the Strain History on TWIP Steel Deformation Mechanisms in the Deep-Drawing Process
}

\author{
R. LAPOVOK, I. TIMOKHINA, A-K. MESTER, M. WEISS, and A. SHEKHTER
}

\begin{abstract}
A study of preferable deformation modes on strain path and strain level in a TWIP steel sheet was performed. Different strain paths were obtained by stretch forming of specimens with various shapes and tensile tests. TEM analysis was performed on samples cut from various locations in the deformed specimens, which had different strain paths and strain levels and the preferable deformation modes were identified. Stresses caused by various strain paths were considered and an analytical analysis performed to identify the preferable deformation modes for the case of single crystal. For a single crystal, in assumption of the absence of lattice rotation, the strain path and the level of accumulated equivalent strain define the preferable deformation mode. For a polycrystalline material, such analytical analysis is not possible due to the large number of grains and, therefore, numerical simulation was employed. For the polycrystalline material, the role of strain path diminishes due to the presence of a large number of grains with random orientations and the effect of accumulated strain becomes dominant. However, at small strains the strain path still defines the level of twinning activity. TEM analysis experimentally confirmed that various deformation modes lead to different deformation strengthening mechanisms.
\end{abstract}

https://doi.org/10.1007/s11661-018-4586-3

(c) The Minerals, Metals \& Materials Society and ASM International 2018

\section{INTRODUCTION}

TWIP steels attract interest globally due to their unique combination of strength (up to $1800 \mathrm{MPa}$ UTS) and ductility (60-100 pct elongation). ${ }^{[1,2]}$ The microstructure of TWIP steel is austenite (fcc) with low stacking fault energy (SFE) between 15 and $40 \mathrm{~mJ} /$ $\mathrm{m}^{2[1]}$ favorable for activation of twinning deformation mode and particular strain hardening behavior. Twin boundaries are effective in blocking dislocation motion, and at the same time they can act as slip planes to accommodate the dislocations. ${ }^{[2]}$ It is widely understood that the balance between slip and twinning activity depends on the stacking fault energy ${ }^{[1]}$ but the orientation dependence of preferable deformation modes is less investigated.

R. LAPOVOK, I. TIMOKHINA, A-K. MESTER, and M. WEISS are with the Institute for Frontier Materials, Deakin University, Waurn Ponds, VIC 3216, Australia. Contact e-mail: r.lapovok@ deakin.edu.au A. SHEKHTER is with the Aerospace Division, Defence Science and Technology, Fisherman's Bend, VIC 3207, Australia.

Manuscript submitted December 5, 2017.

Article published online March 27, 2018
A study on the orientation dependence of deformation mechanisms in TWIP steel has been reported by Karaman et al. ${ }^{[3]}$ using single crystals of Hadfield manganese steel. Tensile samples were deformed in three different crystallographic orientations, $\langle 001\rangle$, $\langle 111\rangle$, and $\langle 123\rangle$. Microscopic observation revealed that in crystals oriented along the [111] direction, twinning is the primary macroscopic deformation mode, while slip governs the macroscopic deformation in the crystals oriented along the [001] direction.

Furthermore, a Hadfield steel single crystal was tested in two deformation modes, namely tension in [001] and [1510] directions and compression in [001], [123], [1111] directions to study the competing mechanisms of slip and twinning. ${ }^{[4]} \mathrm{A}$ model for the twin nucleation stress as a function of the crystallographic orientation and stress direction was developed.

Plastic deformation of a polycrystalline material will start either with slip or twinning in each crystal depending on the orientation of the crystal and the level of shear stress. If the shear stress on a slip system reaches the critical value $\tau_{\text {rss }}^{\mathrm{s}}$ (critical resolved shear stress), slip is initiated. According to Schmid's law, uniaxial tension starts slip when the resolved shear stress on a closed packed plane reaches critical slip stress. ${ }^{[5]}$ The slip directions for fec crystals are $\langle 110\rangle$ within $\{111\}$ planes. 
Twinning in fcc crystals occurs by shear on the (111) plane in the $\langle 11 \overline{2}\rangle$ direction with mirror shifted planes (11) $)$ and reflected direction $\langle 112\rangle,{ }^{[6]}$ Twinning nucleates by Shockley partial dislocations with a Burger vector $\frac{a}{6} 112$ on $\{111\}$ close-packed planes. ${ }^{[7]}$ Therefore, the resolved shear stress on the slip plane needs to exceed a critical value, $\tau_{\mathrm{rss}}^{\mathrm{tw}}$, in order to move the twinning dislocation. As the resolved shear stress can be calculated by the Schmid's law, often the criteria for the dominant deformation mechanism are based on the comparison of the Schmid's factors for slip, $m_{\mathrm{s}}$, and twinning, $m_{\mathrm{tw}}{ }^{[8]}$

For example, in Guttierrez et al. ${ }^{[9]}$ the assumption is made that the critical resolved shear stress for both slip and twinning is approximately equal. In fcc metals, multiple slip is required for twinning to occur. Therefore, once a multiple slip is activated, twinning occurs when the twinning stress $\tau_{\mathrm{rss}}^{\mathrm{tw}}$ is larger than the slip stress $\tau_{\mathrm{rss}}^{\mathrm{s}}$. A condition for preferable twin appearance in the grain is that the Schmid factor for twinning is higher than Schmid factor for slip: $m_{\mathrm{tw}}>m_{\mathrm{s}}$.

For simple deformation modes, Yang et al. ${ }^{[10]}$ and Meng et al. ${ }^{[11]}$ have shown that deformation twinning is favored during tension because the grains rotate toward the $\langle 111\rangle / / \mathrm{TA}$ (TA - tensile axis), which renders the crystals favorably orientated for twinning (high Schmid's factor). Deformation twinning is suppressed during compression because the grains rotate toward the $\langle 101\rangle$ axis, which renders them unfavorably oriented for twinning (small Schmid's factor).

In Guttierrez, ${ }^{[12]}$ texture sharpens during tensile deformation leading to texture components characterized by $\langle 111\rangle / /$ TA and $\langle 001\rangle / /$ TA. The deformation twinning mainly occurs in grains that are oriented close to the $\langle 111\rangle / /$ TA directions and only a small fraction of grains with other orientations contain twins. At higher strain (0.3), the influence of grain orientation on twinning activity decreases significantly. With the exception of $\langle 001\rangle / / \mathrm{TA}$, where angular deviation is less than $5 \mathrm{deg}$, all other orientations contain deformation twins.

For complex deformation modes, the co-yield surfaces in fcc single crystals for slip on $\{111\}\langle 110\rangle$ and twinning on $\{111\}\langle 112\rangle$ have been analyzed for general stress states by Chen et al. ${ }^{[13]}$ using the Bishop-Hill maximum work principle and Kocks representation of the yield surface. ${ }^{[14]}$ The ratio, $\xi$, of critical resolved shear stress of twinning, $\tau_{\mathrm{rss}}^{\mathrm{tw}}$, to critical resolved shear stress of slip, $\tau_{\mathrm{rss}}^{\mathrm{s}}$ has been introduced. It was shown that when $\frac{1}{\sqrt{3}} \leq \xi \leq \frac{2}{\sqrt{3}}$ (within the range of $0.58 \ldots 1.15$ ) slip and twinning can occur simultaneously. However, if $\xi>\frac{2}{\sqrt{3}}$ only slip will be activated, and if $\xi<\frac{1}{\sqrt{3}}$ only twining will be activated.

This rule for $\xi$ values was further illustrated for plane strain compression test of the fcc single and polycrystals in Chen. ${ }^{[15]}$ The fcc cubic crystals under plane strain compression were considered and the influence of $\xi$ on the yield stress states and the selection of active systems for the main ideal orientations of interest was investigated. The results showed that twinning is difficult for the $\{100\}\langle 001\rangle,\{110\}\langle 001\rangle$, and $\{112\}\langle 110\rangle$ orientations, and becomes easier for the $\{110\}\langle 112\rangle,\{112\}\langle 111\rangle$, $\{123\}\langle 634\rangle,\{110\}\langle 111\rangle$, and $\{110\}\langle 110\rangle$ orientations. Compared with pure slip, the yield strength anisotropy decreases with the appearance of twinning. A factor, $M$, based on the Taylor factors was introduced to describe the twinning ability of different orientations for polycrystalline material and simulated for all ranges of $\xi$ using the full constraints Taylor model.

The modeling of complex deformation modes becomes more complicated for the plane stress case, when all three principal strains of the strain tensor, $\varepsilon_{11}, \varepsilon_{22}, \varepsilon_{33}$ can be different from zero and have non-linear history during deformation as in the deep-drawing process. It should be noted that the prediction of the deformation mode in polycrystalline material under complex strain history is only possible using numerical codes, for example, the viscoplastic self-consistent (VPSC) polycrystal code. ${ }^{[16]}$

Several challenges for modeling of TWIP steel deformation behavior using numerical simulation can be listed: (i) deformation twinning is usually studied at relatively small strains and the further lattice rotation of the twin during large plastic deformation is not considered; (ii) possible co-rotation of the mother grain and the twin during deformation induces changes in the favorable deformation mode along the strain history; (iii) It is very difficult to address these issues by analytical models. However, analytical consideration of a single crystal under plane stress conditions can give a clear indication of the dominant deformation mode, especially for small strains. Under the condition that the crystal does not rotate during deformation, the analytical model can be used to predict the possible appearance of twins or the slip mode.

In this paper, a study of the deformation mechanisms for varying strain paths has been performed. These strain paths were obtained in different locations of the TWIP steel samples during the stretch forming of sheets with altered width and y uniaxial tensile test. Analytical modeling of the single crystal response in the absence of lattice rotation and numerical simulation of the polycrystalline TWIP steel sheet in stretch forming and the tensile test, using a special code developed, have been carried out. It should be noted that the drawing process has been chosen as convenient tool to have different series of stress paths and the results are relevant to any forming process.

\section{MATERIAL AND EXPERIMENTAL TECHNIQUES}

Cold-rolled TWIP steel sheet with composition of Fe-18Mn-0.6C-1Al (wt pct) was obtained from POSCO Ltd. (Korea). The microstructure was austenitic with an average grain size of $2.5 \pm 0.2 \mu \mathrm{m}$.

The influence of the strain path on the deformation mechanisms in TWIP steel was investigated using three types of plane strain tests, namely a stretch forming test (Figures 1(a) and (b)) and a tensile test (Figure 1(c)). The stretch forming test was performed on two different 

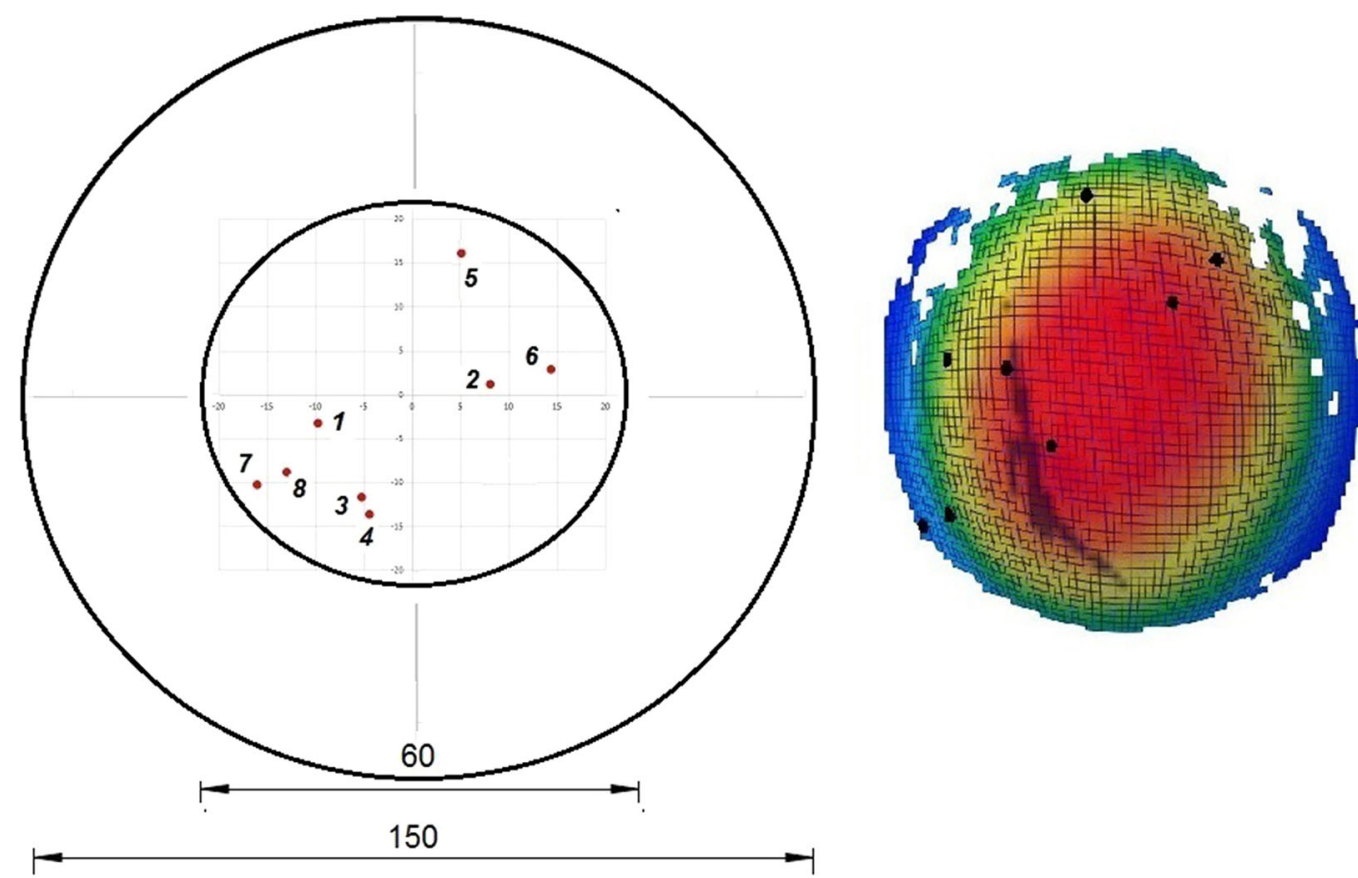

(a)
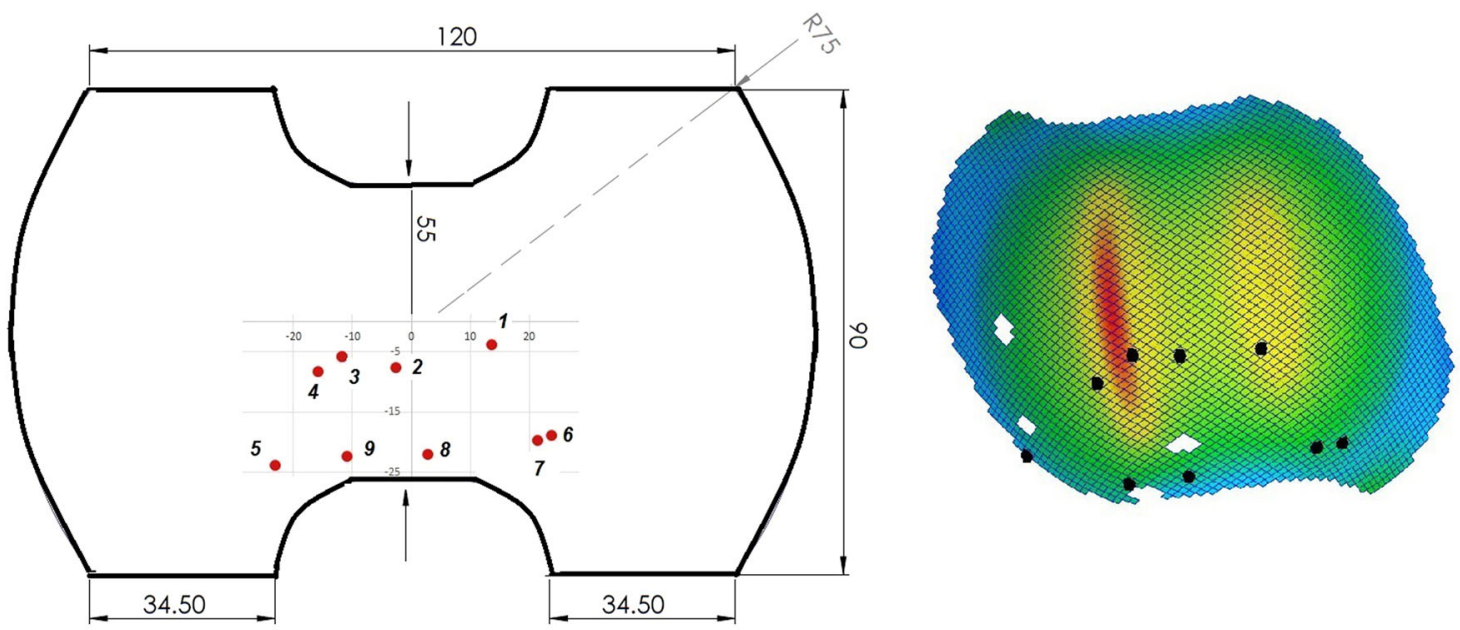

(b)

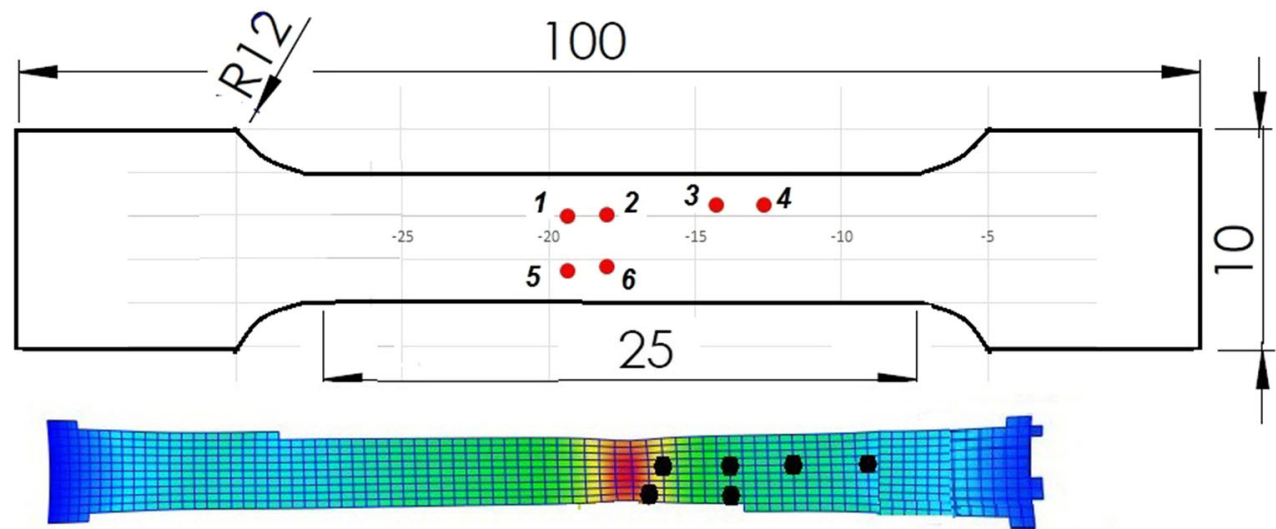

(c)

Fig. 1-Initial sample drawing (drawing not to scale) with position of traced points and traced points position after deformation shown with color contours representing equivalent strain. (a) bi-axial loading, $(b)$ plane strain loading, $(c)$ tensile loading (Color figure online). 


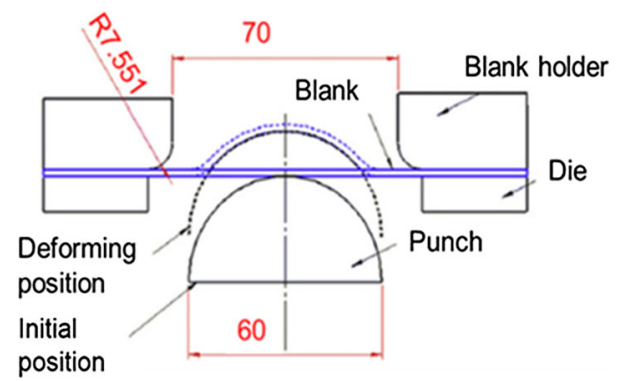

Fig. 2-Stretch forming tool setup.

sample shapes (Figures 1(a) and (b)), giving near-uniaxial, bi-axial, and intermediate stress conditions.

Tensile tests were carried out on an Instron tensile test frame type 8801 equipped with a $100-\mathrm{kN}$ load cell and hydraulic jaws. The tensile samples were water jet cut in rolling direction and dog bone shaped with a gage length of $25 \mathrm{~mm}$ (Figure 1(c)) and a width of $5 \mathrm{~mm}$. Samples were tested at a strain rate of $0.001 \mathrm{~s}^{-1}$ until fracture occurred.

Stretch forming tests over a hemispherical dome punch were performed in an Ericson sheet metal tester (Figure 2) and a maximum blankholder force of $220 \mathrm{KN}$ applied to clamp the sample. To achieve near frictionless conditions, a sandwich construction of three $0.5-\mathrm{mm}$ polypropylene sheets with Milkfat applied in between the layers and on the punch surface was used for lubrication.

The sample shape shown in Figure 1(b) was applied to test for plane strain, while round blanks of $150 \mathrm{~mm}$ diameter (Figure 1(c)) were tested to achieve bi-axial forming conditions. In all tests, a punch speed of $13 \mathrm{~mm} /$ min was used. This produced strain rates between 0.0037 and $0.0049 \mathrm{~s}^{-1}$ for plane strain and bi-axial forming conditions, respectively; this is comparable to the strain rate of $0.001 \mathrm{~s}^{-1}$ tested in the tensile tests.

During the tensile and the stretch forming tests, the strain distribution was analyzed using the optical strain measurement system GOM Aramis. A more detailed description of the GOM Aramis system can be found in Reference 15. Several points for each test with their initial and final position shown in Figures 1(a) through (c) have been selected and their strain path, characterized by the three principal strains, recorded.

Transmission Electron Microscopy (TEM) analysis was performed on the deformed samples cut from the traced points marked as 1, 2, etc. in Figure 3. The EDM cutting was used to avoid formation of additional microstructural features during sample preparation. Then, the samples from the outer surface were electro-polished with 5 pct of perchloric acid in methanol using a twin-jet Tenupol unit, operating at $50 \mathrm{~V}$ and a temperature of $-25^{\circ} \mathrm{C}$. TEM was performed on a Philips CM 20, at $200 \mathrm{kV}$.

\section{RESULTS AND DISCUSSION}

\section{A. Analysis of the Strain Path}

In Figure 4, the strain history is presented as major $\varepsilon_{11}$ strain $v s$ minor $\varepsilon_{33}$ strain (Figure $4(\mathrm{a})$ ) and equivalent

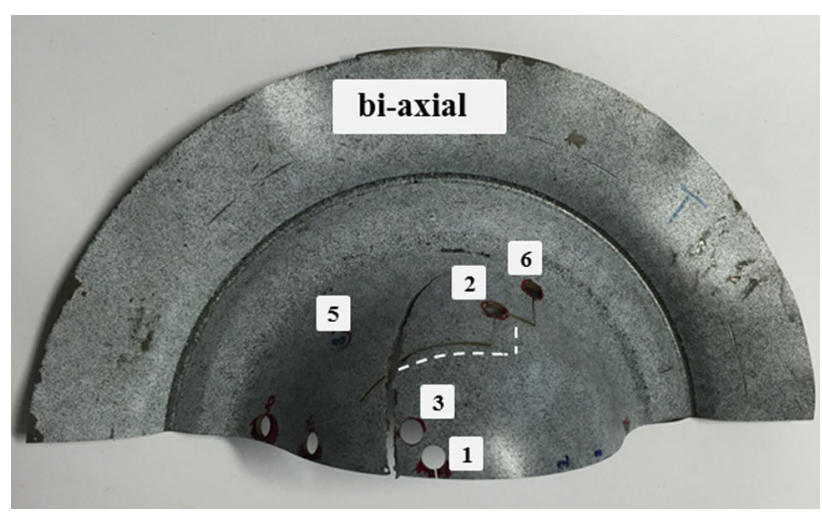

Fig. 3-Representative sample after bi-axial loading with positions of traced points $(1,2,3,5,6)$ used for TEM sample preparation (dotted line represents wire-cutting line).

strain $v$ s time (Figure 4(b)) for the bi-axial loading case. It can be seen that not only the level of equivalent strain is different in these points but also that the strain history varies significantly for all these points. For example, for points $1,3,4,5$ both principal strains increase uniformly keeping approximately the bi-axial strain state, while for points $2,6,7,8$ the major strain increased two-to-three times quicker compared to the minor strain.

The strain history for plain strain case is presented as major $\varepsilon_{11}$ strain vs minor $\varepsilon_{33}$ strain (Figure 5(a)) and equivalent strain vs time (Figure 5(b)). These points can be divided in two groups, points $1,2,3,4$, for which the strain history is initially similar to bi-axial loading and later changes to plane strain, and points $5,6,7,8,9$, where the strain increases in plane strain mode with a positive major strain and a negative minor strain. The level of equivalent strain is lower (Figure 5(b)) for group 5-9 compared to group 1-4.

The strain history during tensile testing is presented as major $\varepsilon_{11}$ strain $v s$ minor $\varepsilon_{33}$ strain and equivalent strain $v s$ time for the uniaxial forming case, in Figures 6(a) and (b), respectively. It can be seen that the closer position of the tracked points to the middle of sample the larger is the accumulated equivalent strain and both major and minor strains. Otherwise, all points except 5 have the same strain history. Initially material deformation behavior in all test locations was near-uniaxial strain. While sample points 2, 3, 4, and 6 show very similar levels of equivalent strain at the end of the test, in test locations 1 and 5 a rapid increase in equivalent strain is observed, which is indicative of the localization of strain and the development of local necking.

\section{B. Theoretical Analysis of the Deformation Mode for a Cube-Oriented Single Crystal}

To understand dependence of the predominant strain mechanism on the strain path, a simple case of one single crystal was considered as illustrated in Figure 7.

For complex loading paths, slip begins when the resolved slip shear stress on the $\{111\}\langle 110\rangle$ slip systems reaches the critical slip shear stress, $\tau_{\mathrm{rss}}^{\mathrm{s}}$ :

$$
\sigma_{i} n_{i} d_{i}= \pm \tau_{\mathrm{rss}}^{\mathrm{s}} \quad(i=1,2,3) .
$$




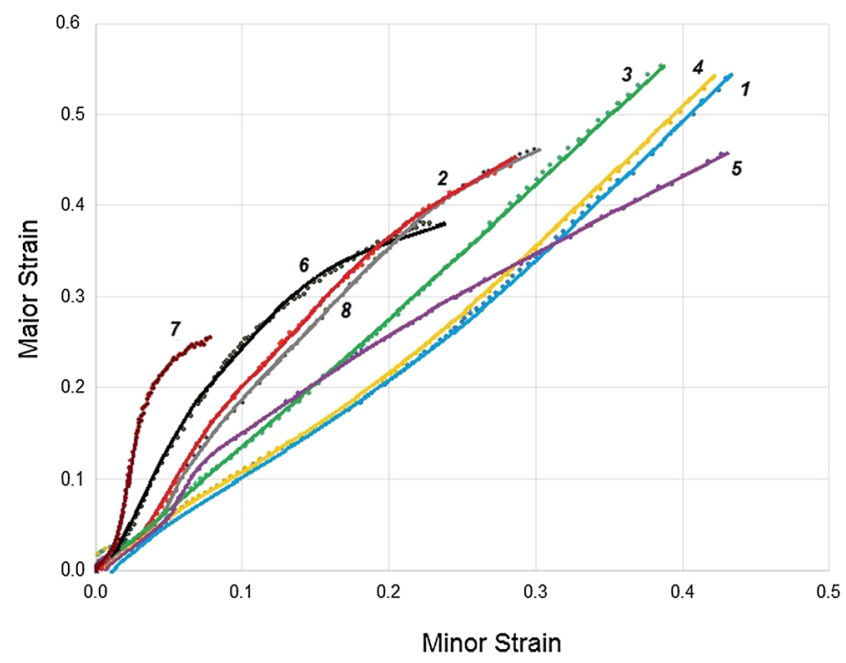

(a)

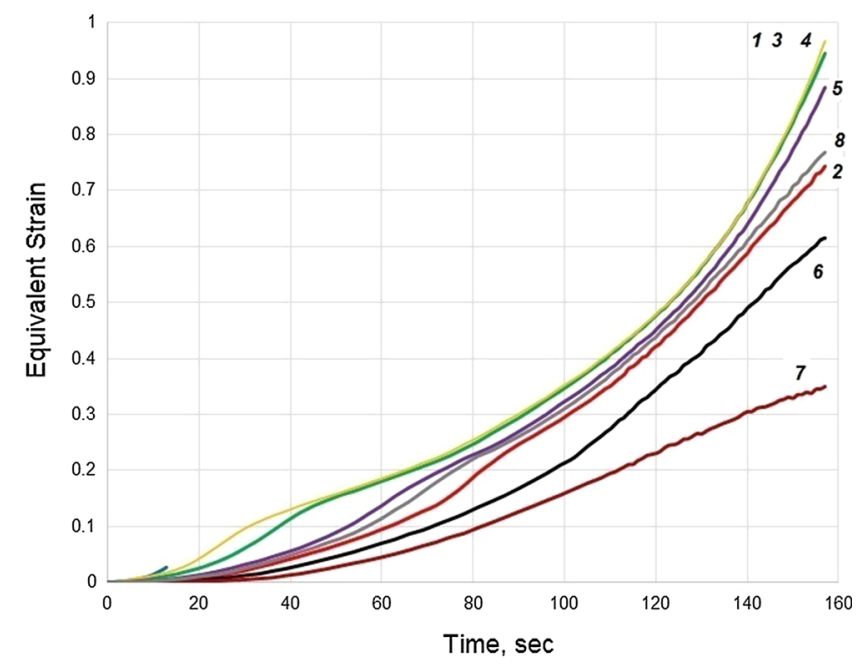

(b)

Fig. 4-Experimental strain measurements obtained during bi-axial loading in eight points of the sample shown in Fig. 1(a). (a) Major strain vs minor strain, (b) equivalent strain.

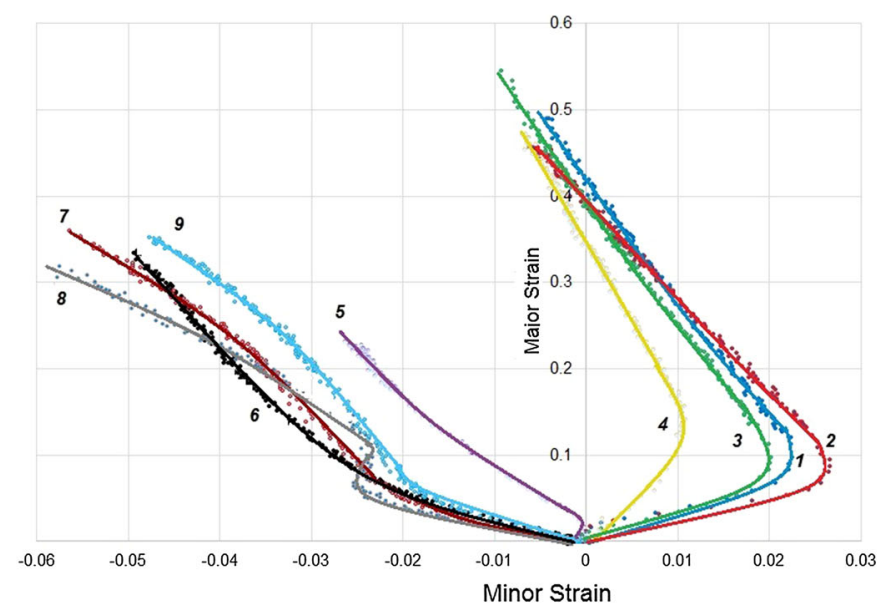

(a)

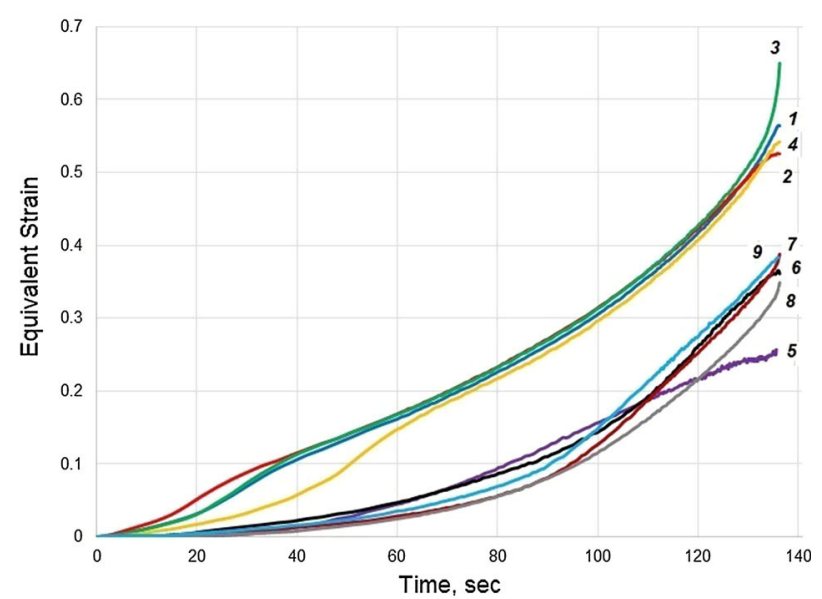

(b)

Fig. 5-Experimental strain measurements obtained during plane strain loading in nine points of the sample shown in Fig. 1(b). (a) Major strain $v s$ minor strain, $(b)$ equivalent strain.

Here $n$ is normal to the slip planes $\{111\}$ and $d$ is slip direction, and they are both unit vectors. The $n_{i} d_{j}$ product is the generalized Schmid factors. ${ }^{[11]}$

Assuming that the stress tensor only contains diagonal components that are non-zero, that is, we are in the reference system of the principal stresses, the three components of the stress tensor will be named $\sigma_{1}, \sigma_{2}, \sigma_{3}$. Note that there is no stress perpendicular to the sheet: $\sigma_{3}=0$. It is further assumed that the [100], [010], [001] directions of the fcc crystal coincide with the principal axis of stress, as shown in Figure 7.

Considering all 24 slip systems, one can show that they can be divided into three equivalent groups with shear stress in a $\{111\}\langle 110\rangle$ slip direction system:

$$
\tau_{\mathrm{rss}}^{\mathrm{s}}= \pm \frac{1}{\sqrt{6}}\left(\sigma_{i}-\sigma_{j}\right) \quad i, j=1,2,3
$$

Similar to Schmid's law for slip, Eq. [1] can be used to obtain the shear stress in the twinning direction $\langle 11 \overline{2}\rangle$ on the $\{111\}$ twinning planes. Twinning occurs if the resolved twinning shear stress reaches a critical stress, $\tau_{\mathrm{rss}}^{\mathrm{tw}}$ :

$$
\sigma_{i} n_{i} t_{i}=\tau_{\mathrm{rss}}^{\mathrm{tw}}, \quad(\mathrm{i}=1,2,3) .
$$

Here $\boldsymbol{n}$ is normal to the $\{111\}$ twin planes and $\boldsymbol{t}$ is the twin direction.

Considering 12 twinning systems, we can divide them, similarly as in Reference 13, into four equivalent groups with the following twinning resolved shear stresses:

$$
\tau_{\mathrm{rss}}^{\mathrm{tw}}=\frac{1}{\sqrt{18}}\left(\left(\sigma_{i}-\sigma_{j}\right)+\left(\sigma_{k}-\sigma_{j}\right)\right) \quad i, j, k=1,2,3 .
$$


The $\xi$ ratio $^{[11]}$ for the crystal under loading shown in Figure 7 is then given by

$$
\xi=\frac{\tau_{\mathrm{rss}}^{\mathrm{tw}}}{\tau_{\mathrm{rss}}^{\mathrm{s}}}= \pm \frac{1}{\sqrt{3}} \frac{\left(\left(\sigma_{i}-\sigma_{j}\right)+\left(\sigma_{k}-\sigma_{j}\right)\right)}{\left(\sigma_{i}-\sigma_{k}\right)} \quad(i, j, k=1,2,3),
$$

which should be in the range $\left(\frac{1}{\sqrt{3}} \cdots \frac{2}{\sqrt{3}}\right)$ to have both deformation mechanisms activated. It ${ }^{2}$ can be seen that the $\xi$ ratio is defined by the maximum shear stresses on planes bisecting the principal directions. Defining the ratio of shear stresses in expression [5] as $\mathrm{R}$, we establish that slip and twinning can occur together when $1<R<2$. For $R>2$, only twinning will be activated, and for $R<1$ only slip will be activated.

The time history of the $R$ ratio for the selected cube orientation is presented for all points in Figure 8. Note that this is computed analytically for a single crystal with its sides coinciding with the principal directions of the strain stress tensor and in assumption that the lattice rotation about the principal axes of the strain tensor can be neglected. This ratio is shown graphically for the selected point locations in Figure 8 for all three tests.

For those points that were part of the TEM study (Section III-B), the trend lines are shown. Those are points 3, 5, 6, 8 in bi-axial; points $2,3,6,9$ in plane strain; and points 1, 3 of the tensile test. The dashed lines in Figures 8(a) through (c) separate areas of "twinning only," "twinning and slip," and "slip only." It can be seen that for crystals that coincide with the principal directions of the stress tensor, especially at the beginning of loading, it is expected to observe (i) twinning and slip for points 6,8 , and slip only for points 3, 5 in the bi-axial test; (ii) twinning and slip for the plane strain test; and (iii) dominant twinning for the tensile test.

However, analytical calculations become difficult even for a single crystal, when all 24 slip systems and 12 twinning systems are accounted for, and cannot be carried out for polycrystalline material consisting of many grains with different orientations. The assumption of minimum rotation of the crystals about principal directions of the strain tensor is quite weak. Moreover, any twinning results in rotation of part of the crystal and reorientation with respect to principal directions. Even though simple analytical calculations for a single crystal provided some indication of the dominant deformation mode, modeling using an extended version of the viscoplastic self-consistent (VPSC) polycrystal code, developed in Reference 17, was necessary for analysis of deformation mode in polycrystalline material used in experiment. Results are discussed in the next section.

\section{Modeling of the Deformation Mode for a Polycrystalline Material}

The viscoplastic self-consistent (VPSC) polycrystal code used here considers an 'isotropic' code, because the interaction between a given grain and the homogeneous medium is calculated using the assumption that the medium itself is isotropic. This assumption can be applied to cubic materials with random texture as was the case here for annealed TWIP steel.

To account for twinning, the volume transfer technique was used, which was shown to be a reliable approach. ${ }^{[18,19]}$ This twinning scheme was implemented into the Metz-VPSC code. In the volume transfer scheme, each twin variant is considered as a separate grain created at the beginning of the simulation with zero initial volume fraction. As deformation progresses during the modeling simulation, the volume fraction is transferred from the parent grain to its corresponding twins. This approach can only be applied if the twin orientation relation does not deviate too much from the exact one. A certain deviation angle can be permitted because local dislocation mechanisms, which make the twin grow, can accommodate a small difference. In the present work, the volume transferred from the parent grain to a twin was reduced progressively with the orientation difference, using a linear function. When the orientation difference became larger than $10 \mathrm{deg}$, the volume transfer procedure was stopped.

Except for a direct reorientation of twinned volume, twinning also influences the texture evolution due to a strong latent hardening effect. ${ }^{[20]}$ The twins were assumed to be harder than the parent grains, with initial values twice higher for slip system strengths in the twins with respect to their parent grains. 500 parent grains were considered for the simulation, each with its 12 predefined twins, so the total grain population was composed of 6500 grains. Strain hardening was simulated using the self and latent hardening approach proposed in Reference 21 with four hardening parameters defined from experimental stress-strain curves.

The twin-to-slip ratios and twin volume fractions calculated by the VPSC code are presented in Figure 9 for bi-axial, plane strain, and tensile tests vs equivalent strain obtained from simulations. The level of equivalent strain is close to experimental results presented in Figures 4(b) through 6(b).

It can be seen that the twin-to-slip ratio for polycrystalline material in bi-axial test is not significantly different between the 8 points, though the twin volume fraction is different and depends on the equivalent strain accumulated by the point. The twinning activity drops rapidly for equivalent strains below 0.1 , Figures 9(a) and (b).

It can be noticed that more variation is observed for polycrystalline material in the plane strain test, Figures 9(c) and (d). The group of points marked as 1, 2, 3, 4 has the same tendency as the points in bi-axial test with a sharp decrease in twinning activities and similar values for the twin-to-slip ratio. Another group of points $(6,7,8,9)$ have a twice as high twin-to-slip ratio that reduces with equivalent strain. This group is closer to results for the simulated tensile test results, which are shown in Figures 9(e) and (f). The highest twinning activity can be observed in the necking area, point 1 , and the volume fraction of twins depends on the accumulated equivalent strain.

The results obtained by the polycrystal simulation show the following main trends: 


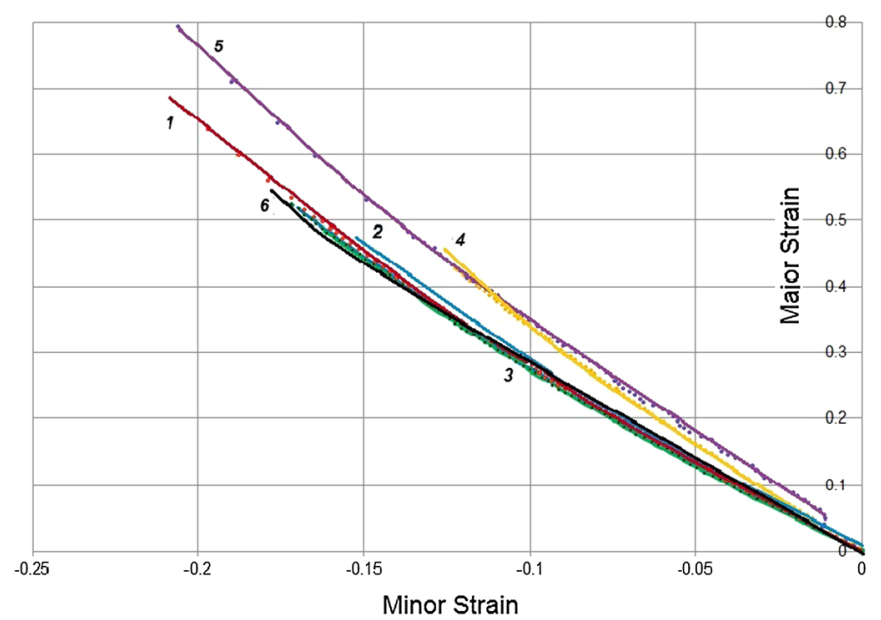

(a)

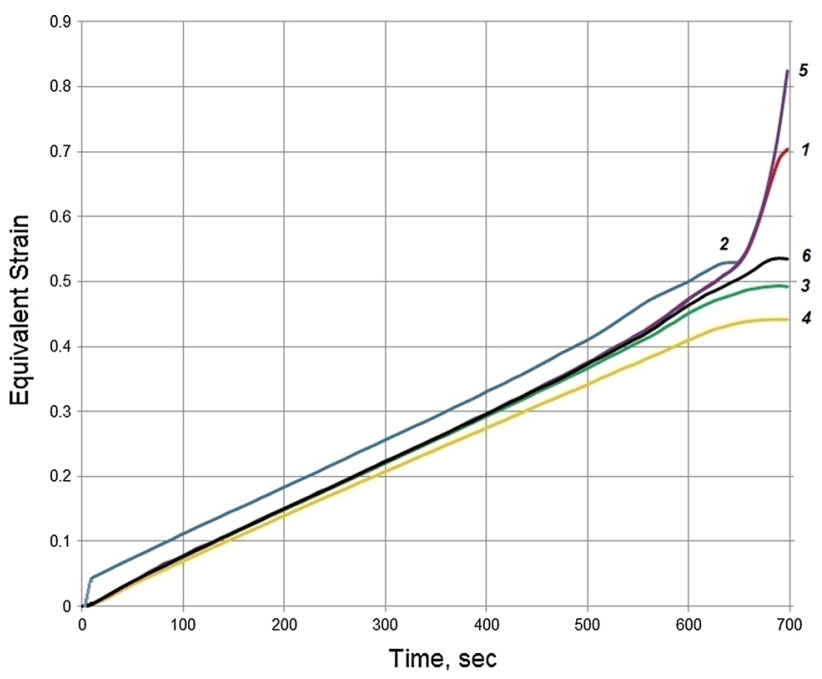

(b)

Fig. 6-Experimental strain measurements obtained during uniaxial strain loading (tensile test) in six points of the sample shown in Fig. 1(c). (a) Major strain $v s$ minor strain, (b) equivalent strain.

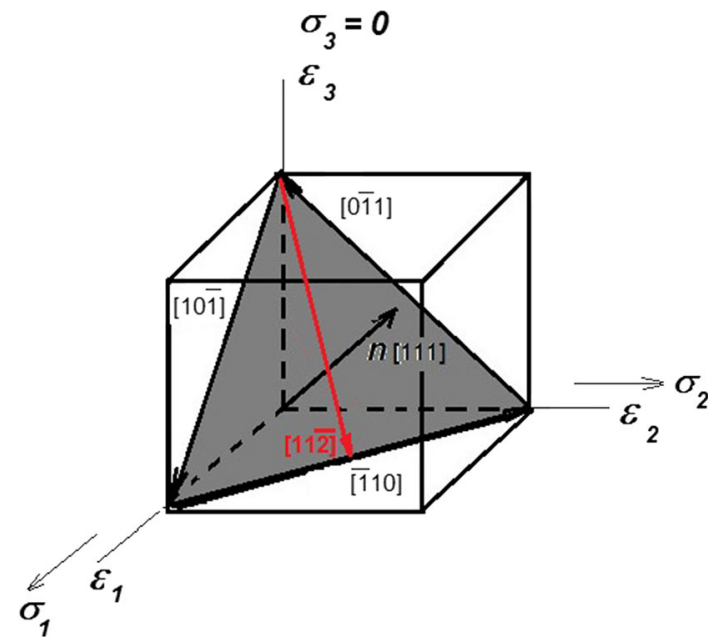

Fig. 7-Crystal orientation with respect to principal directions of the stress (strain) tensor considered for the illustration of the analytical approach.

- The twinning activity is highest in tensile deformation, followed by the plane strain case. The lowest twinning volumes were obtained in bi-axial testing.

- The material is deforming by twinning very heavily in the initial stage of deformation, and then the contribution of twinning to the deformation decreases drastically. This is due to differences between the lattice rotations of the mother and its twin grains; the twins rotate out from their exact twinning positions during large plastic strains.
The above predictions are confirmed by TEM observations that are presented in the next section.

\section{TEM Characterization of the Samples After Different Loading Tests}

TEM study of samples from representative traced points was used to reveal the preferential deformation mechanisms after different strain paths in order to correlate it with the numerical model. The TEM observation was performed on 30-40 austenitic grains for each sample. Theoretically, the SFE for the studied steel should be 14 to $29 \mathrm{~mJ} / \mathrm{m}^{2}{ }^{[22]}$ This $\mathrm{SFE}$ value should promote deformation by twinning ${ }^{[1]}$ regardless of the strain path. However, only TEM samples after tensile loading showed the preferential development of deformation twinning with an increase in equivalent strain compared to the other loading histories. For example, the twin-to-slip ratio for point 1 yielded the highest value of 0.8 compared to all other tests and strain paths and the twin volume fraction was also very high and equal to 0.5, Figures 9(e) and (f).

TEM study after tensile loading was carried out in traced points 1 and 3 named thereafter samples T1 and T3, Figure 10. The T1 sample, near the area of failure, showed that most of the austenite grains have deformation twins or even two sets of twins with different orientations, Figures 10(a) and (b), and micro-twins formed inside the twin planes, Figure 10(c). However, some of the grains revealed evidence of deformation bands that crossed the grain interior and dislocation 


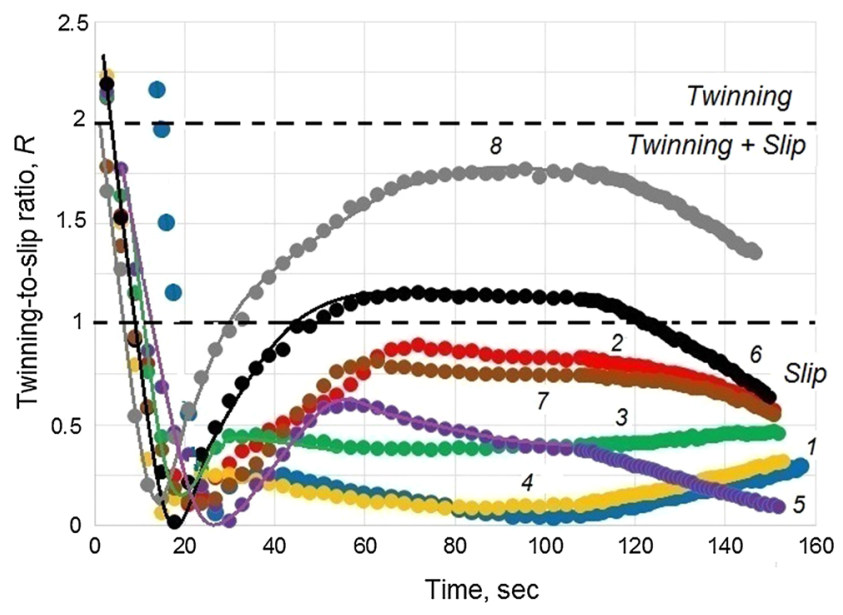

(a)

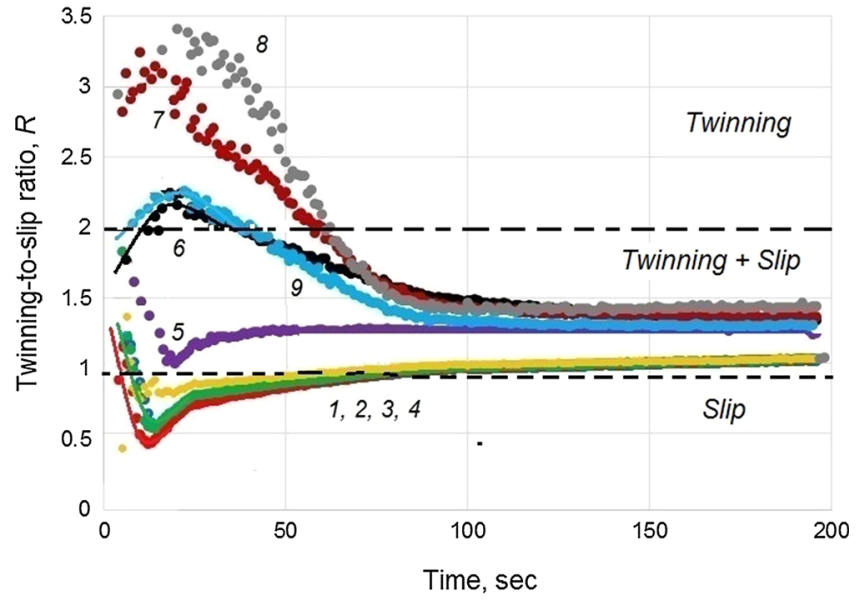

(b)

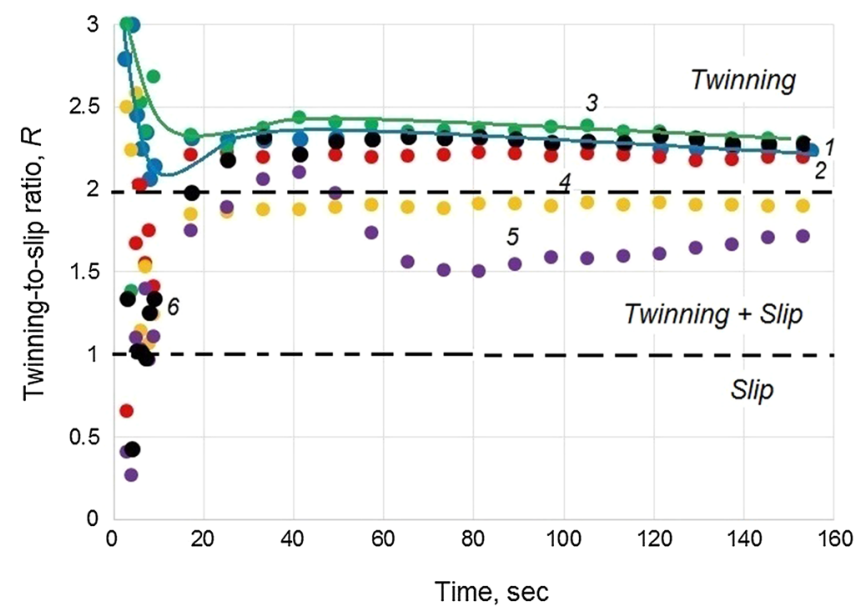

(c)

Fig. 8-Twinning-to-slip ratio $\mathrm{R} v s$ time graphs for the traced points in (a) bi-axial test, $(b)$ plane strain test, $(c)$ tensile test.

pile-up along the twins, Figure 10(d). Point 3 is located at some distance from the necking area and the level of equivalent strain is lower. The difference between the microstructures of samples T1 and T3 is manifested in the number of grains, which exposed the discussed above micro-features. The sample T3 showed decreased number of these grains.

For the bi-axial loading, the traced points, Figure 4, could be divided into two groups: (i) points 2, 6, 7, and 8
(Group 1-non-linear strain path) and (ii) points 1, 3, 4, and 5 (Group 2-almost linear strain path). Two most representative TEM samples from each group: points 6 and 8 (Group 1) and points 3 and 5 (Group 2) were chosen for the TEM study of the microstructure and are labeled as B6, B8 and B3, B5, respectively, hereafter.

The representative microstructural features of the B3 and B5 (Group 2) samples were indicative of the formation of dislocation pile-ups, dislocation walls, 


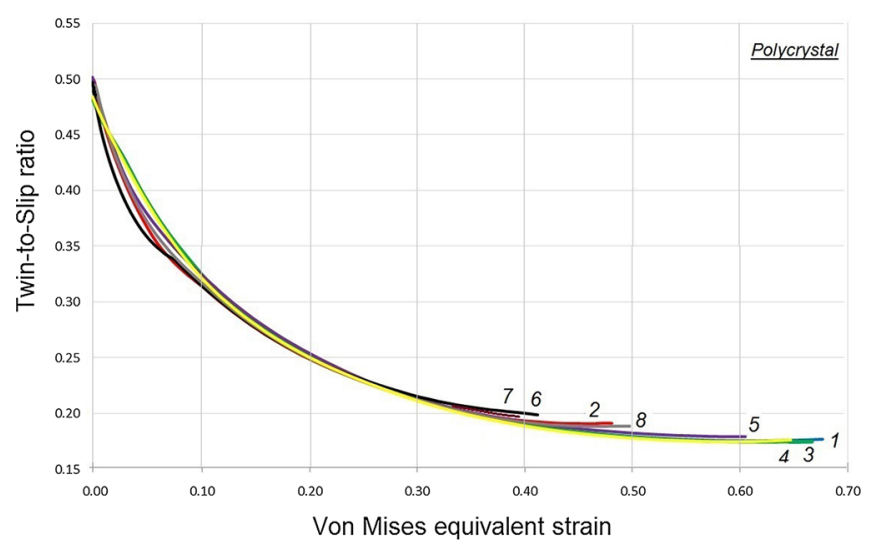

(a)

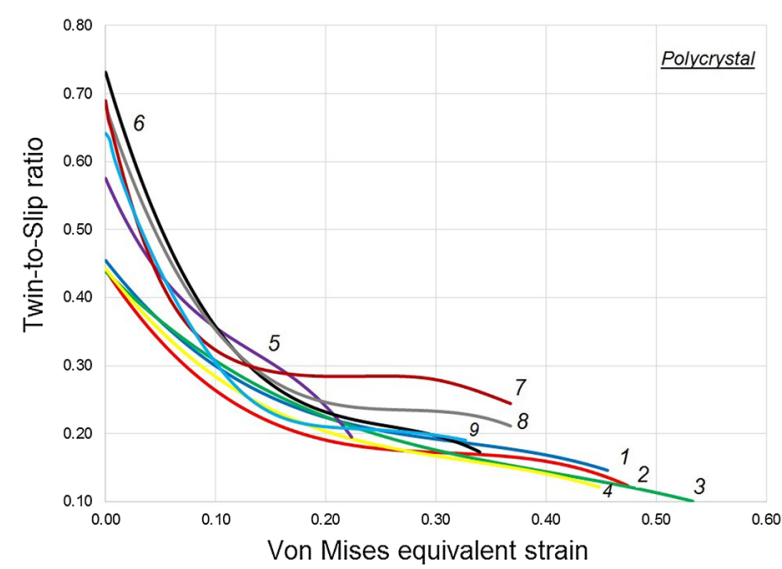

(c)

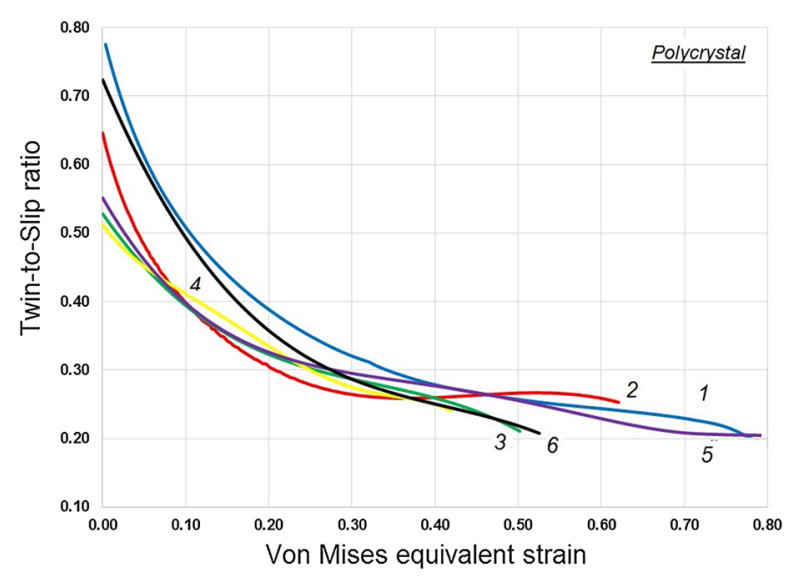

(e)

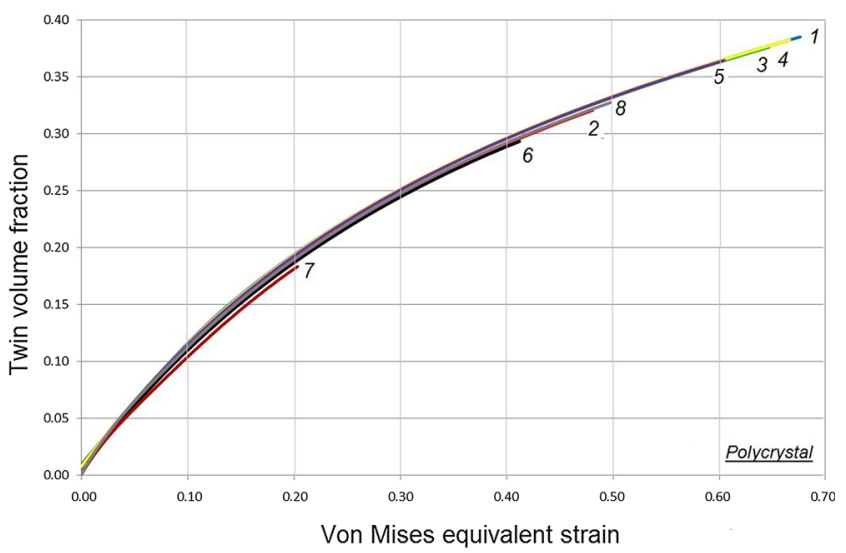

(b)

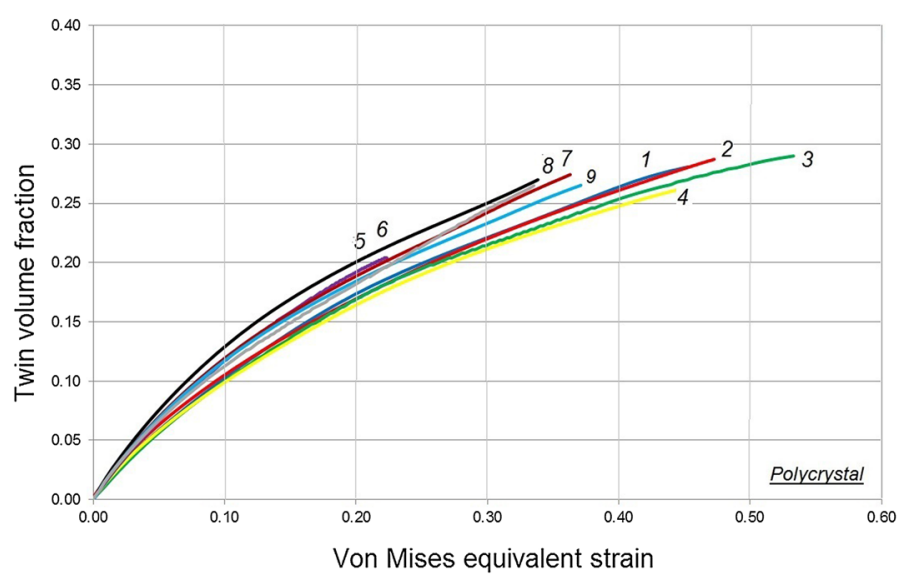

(d)

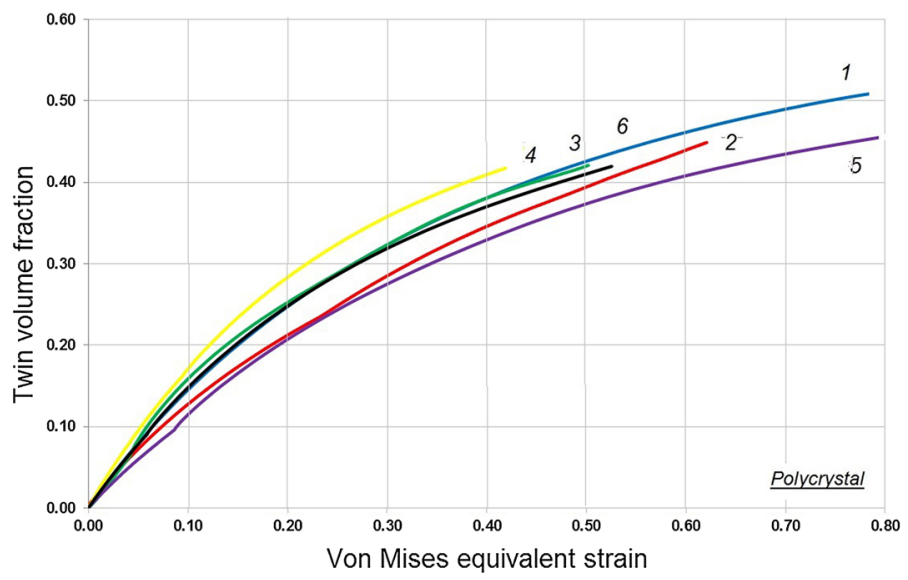

(f)

Fig. 9-Simulation results obtained for a polycrystalline material (500 mother and 6000 twin grains) $(a),(b)$ bi-axial test; $(c)$, $(d)$ plane strain test; $(e),(f)$ tensile test (a, c, e-twin-to-slip ratio; b, d, f-twin volume fraction).

and cells, Figure 11. Only few grains revealed formation of twins in B3, Figures 11(c) and (d)), whereas in B5, twinning was not observed at all. Based on the analysis of the microstructures, it could be concluded that preferential deformation mechanism in these traced points is slip, Figure 11. The twin-to-slip ratio for the bi-axial test is the lowest and it drops rapidly with increasing equivalent strain. Therefore, the number of grains with twins is expected to be small.

The TEM of the B6 sample (Group 1) showed the formation of stacking faults as a predominant microstructural feature, Figure 12. The stacking faults nucleated at the grain boundary as well as in the grain interior, Figures 12(a) through (c). Moreover, some of 


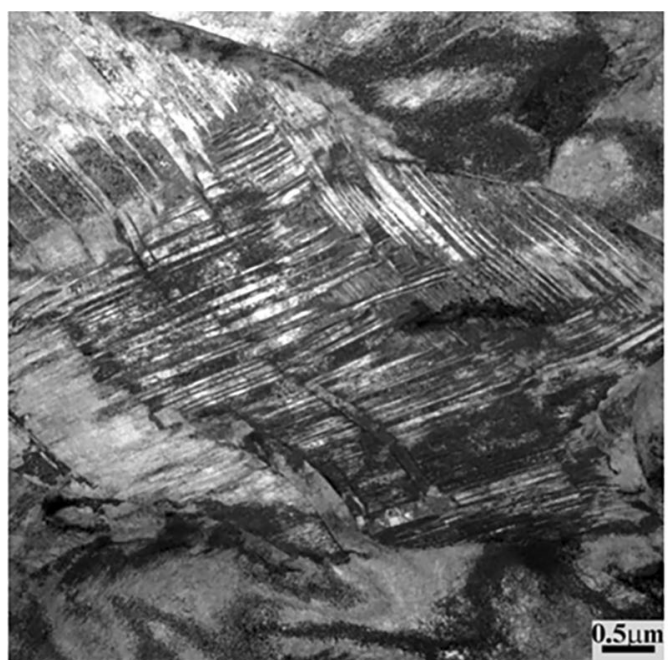

(a)

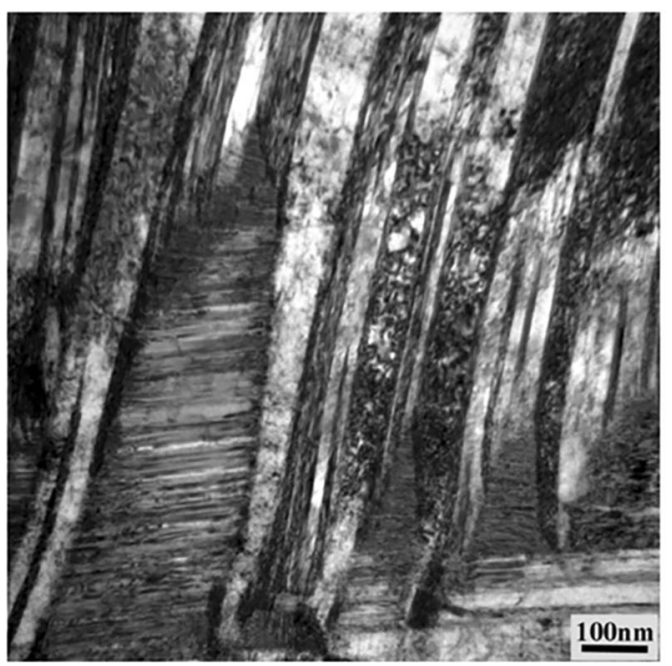

(c)

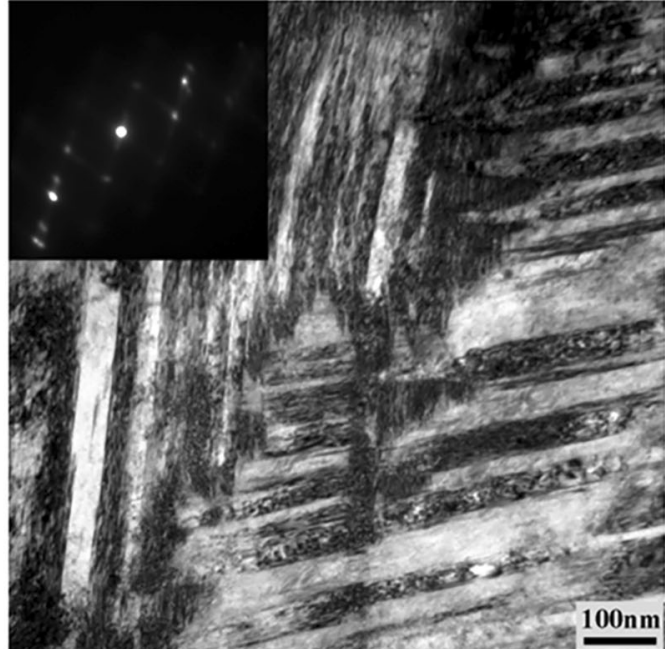

(b)

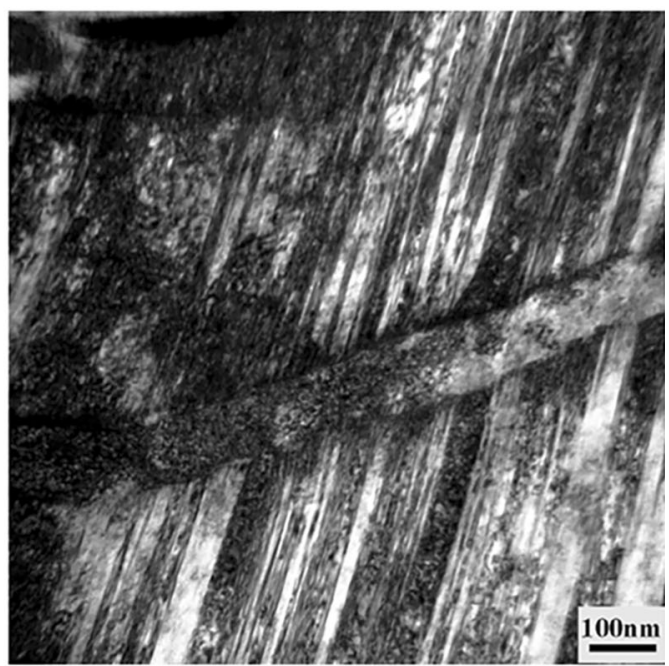

(d)

Fig. 10-Representative TEM images of the deformation substructure formed in samples T1 and T3. (a), (b) Two sets of deformation twins (T1), diffraction pattern of $[110]$, from two sets of twins; $(c)$ micro-twins within the twinning plane (T3); $(d)$ interaction between the twins, deformation bands, and dislocations (T3).

the grains showed stacking faults with different Burgers vectors, which can significantly increase the interaction of dislocations with stacking faults during straining, Figure 12(d). As was reported earlier, ${ }^{[22]}$ a mechanism between stacking faults and dislocations could be the main contributing factor in the work-hardening enhancement of the TWIP steel. Although deformation twinning was not the predominant deformation mechanism, twins were observed in some austenite grains and showed interaction with the dislocations, Figures 12(e) and (f). Hence, the preferential deformation mechanism for this sample was the formation of stacking faults with strengthening being mainly the result of the interaction between stacking faults/twins and dislocations formed due to the plastic deformation.

Contrary to sample B6, the sample B8 (Group 1) after bi-axial loading showed the formation of thick deformation twins as a representative microstructural feature, Figures 13(a) and (b). Certain twins had formations of the secondary micro-twins or stacking faults inside the twinned plane, Figure 13(b). Moreover, it was observed that dislocations formed in the B8 sample tended to form cell structures with thick dislocation walls and dislocation-free areas in the cell interior, Figure 13(c). Only a few austenite grains exposed the formation of thin deformation twins that interacted with the dislocations, Figure 13(d).

It appears that the interaction of dislocations with stacking faults in the B6 sample is related to a more pronounced non-linearity of the strain path, whereas interactions of dislocations with twins as in B8 sample is related to less pronounced non-linearity of strain path even with the presence of secondary micro-twins, Figure 4(a). The non-linearity of strain path can be attributed to anisotropy or strain hardening. ${ }^{[23]}$

The experimental results of the plane strain test showed significant differences between the strain history at points 1, 2, 3, 4 (Group 1) and points 5, 6, 7, 8, 9 


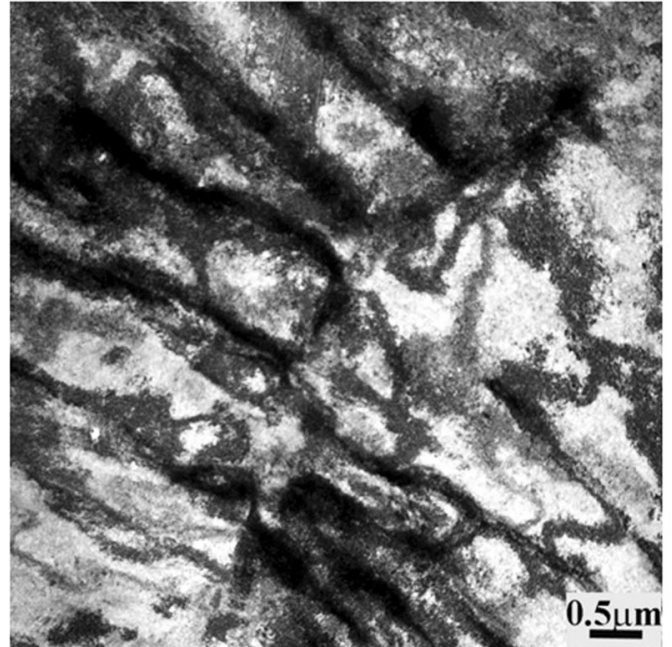

(a)

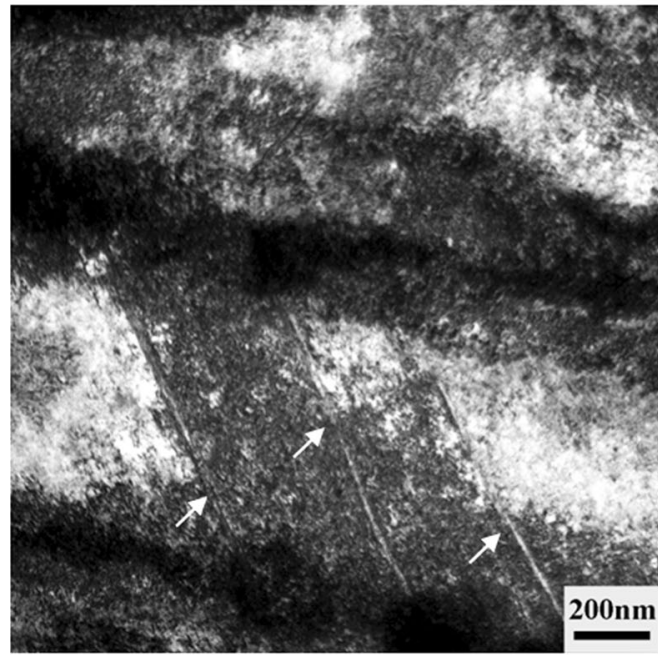

(c)

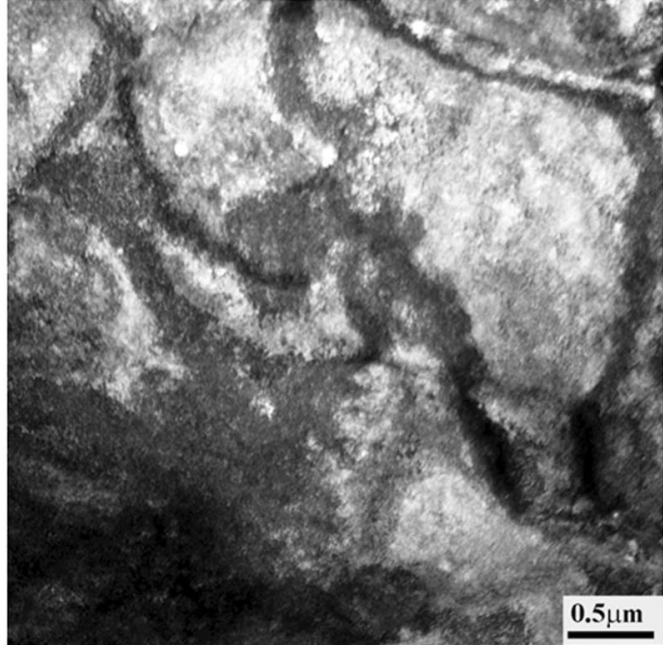

(b)

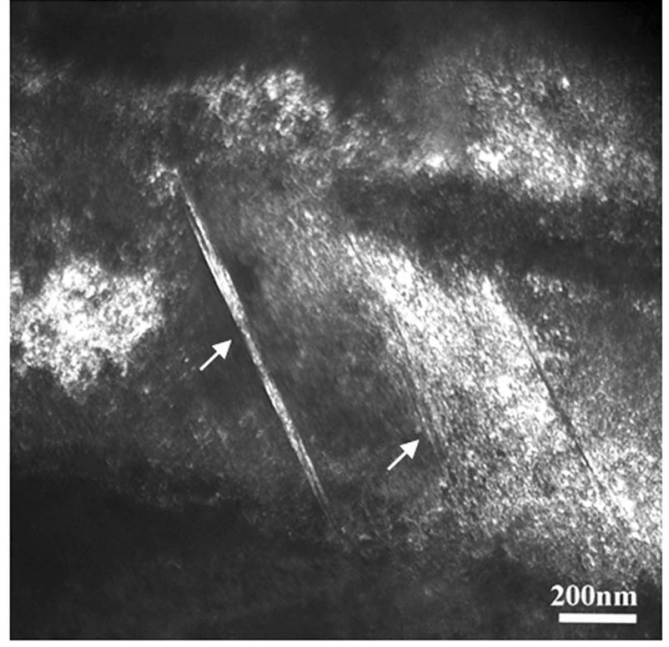

(d)

Fig. 11-TEM micrographs of microstructural features formed in the samples after bi-axial loading. (a) Dislocation pile-ups and cells formed in B3; (b) in B5; (c) bright-field TEM image, and (d) dark-field image of mechanical twins formed in B3.

(Group 2). The strain path of the Group 1 points is monotonic and looks similar to uniaxial strain path, while the strain path of the points belonging to Group 2 initially develops similar to bi-axial loading strain path and then after passing the corner point changes suddenly to the strain path typical for uniaxial loading.

The microstructural features of the samples within each group clearly showed similarities, while the microstructural features of the samples between the groups were different. The representative samples named thereafter P2, P3 (Group 1) and P6, P9 (Group 2) were chosen to characterize the microstructural similarities and differences.

The main microstructural difference between the samples in Group 1 and Group 2 was the formation of secondary twins in Group 2, while in the Group 1 only primary twins were observed. This suggests that the formation of twins with different orientations (i.e., secondary twins) is due to the non-monotonic strain path.
Despite the similarities in the microstructural behavior between samples in Group 1, each sample (P2 and P3) had certain individual features. For example, the presence of deformation bands was observed in the P2 sample, Figure 14(a). The formation of dislocation pile-ups and their interaction with twins was also the microstructural feature of this sample P2, Figure 14(b). In contrast, the P3 sample exposed a lot of austenitic grains with the formation of formed dislocations and dislocation cells and only few of them showed deformation twins, Figures 14(c) and (d). It appeared that these microstructural differences are related to the differences in strain behavior between samples P2 and P3.

The predominant deformation mechanism responsible for the strain behavior of the P6, P9 samples (Group 2), as mentioned above, were the formation of two sets of deformation twins, Figure 15(a), the formation of dislocation pile-ups, and the interaction between twins and dislocations, Figure 15(b). 


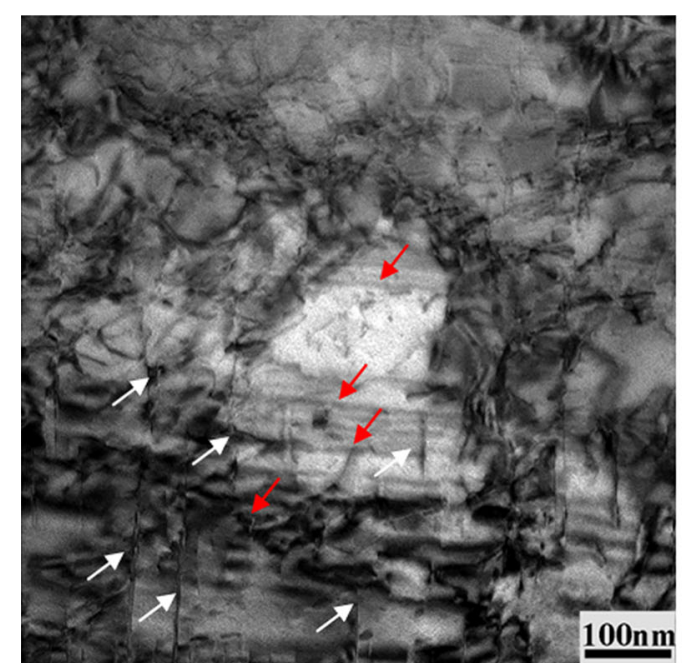

(a)

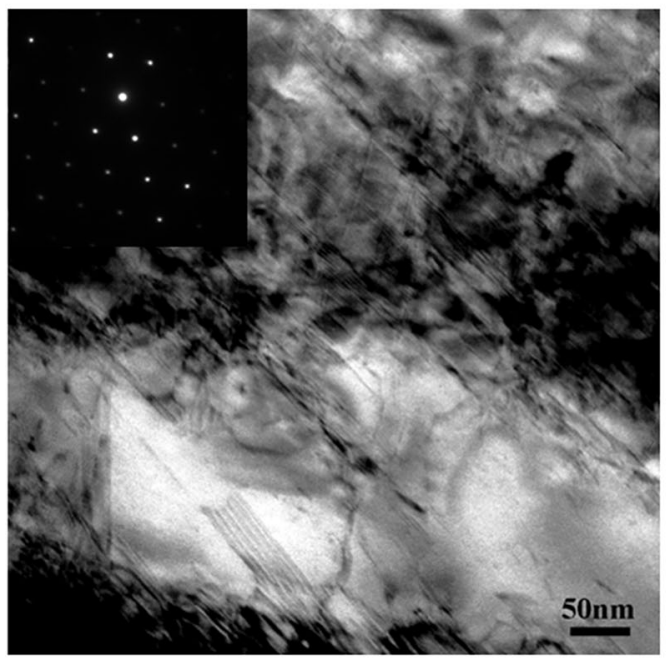

(c)

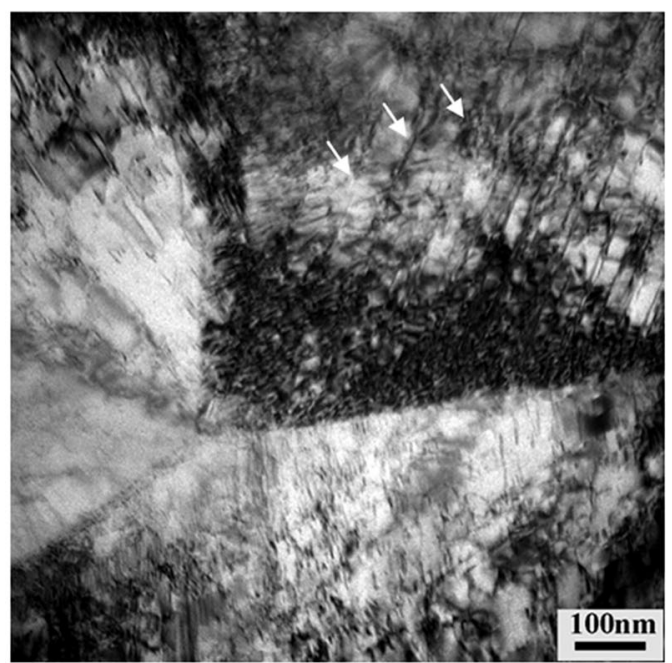

(e)

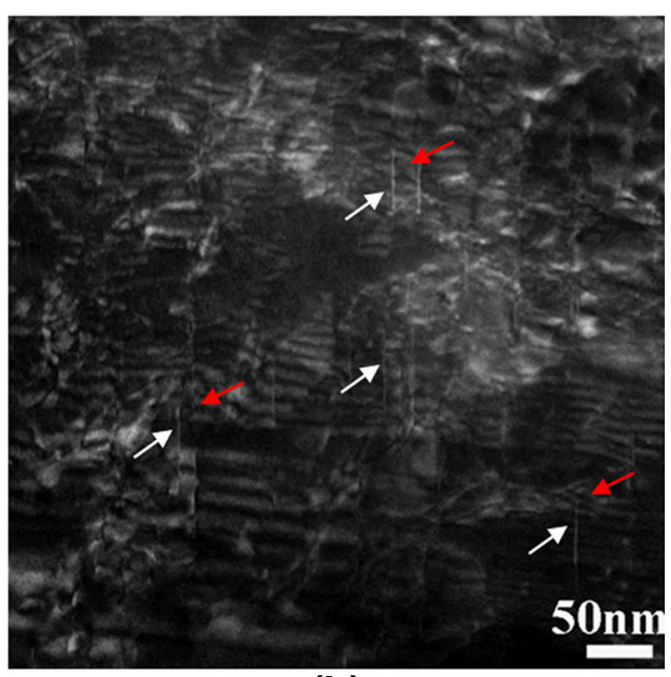

(b)

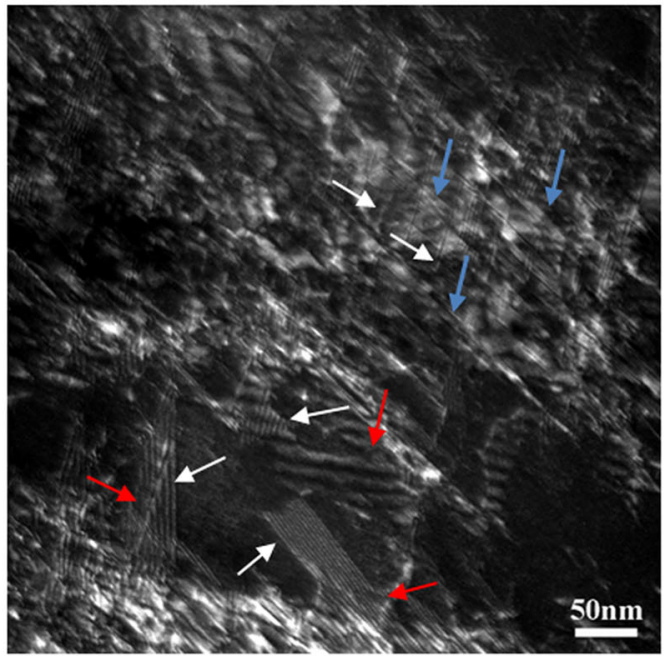

(d)

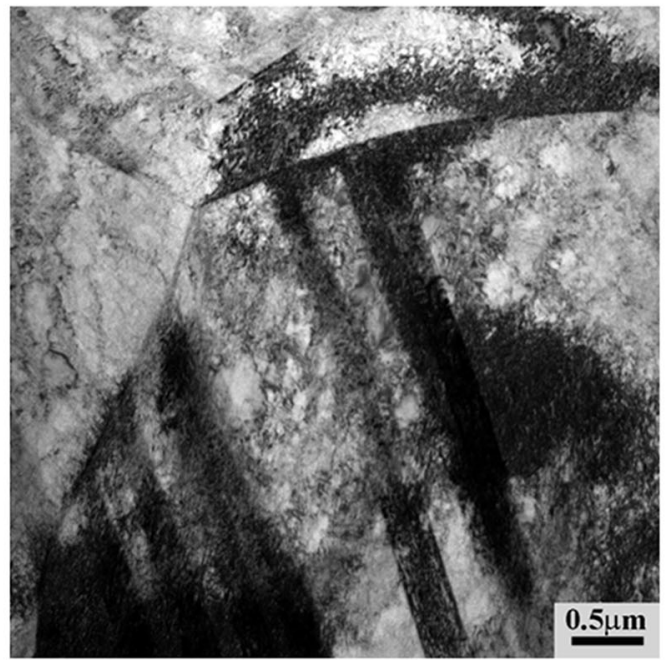

(f)

Fig. 12-TEM micrographs of the microstructural features in sample B6 after bi-axial loading. (a), (b) Stacking faults (white arrows) and dislocations (red arrows) interaction; $(c),(d)$ stacking faults with different Burgers vectors (white and blue arrows) interact with dislocations (red arrows), diffraction patent from grain with the zone axis of $[110]_{\gamma}$; (a, c, e, f—bright field; b, d-dark field) (Color figure online). 


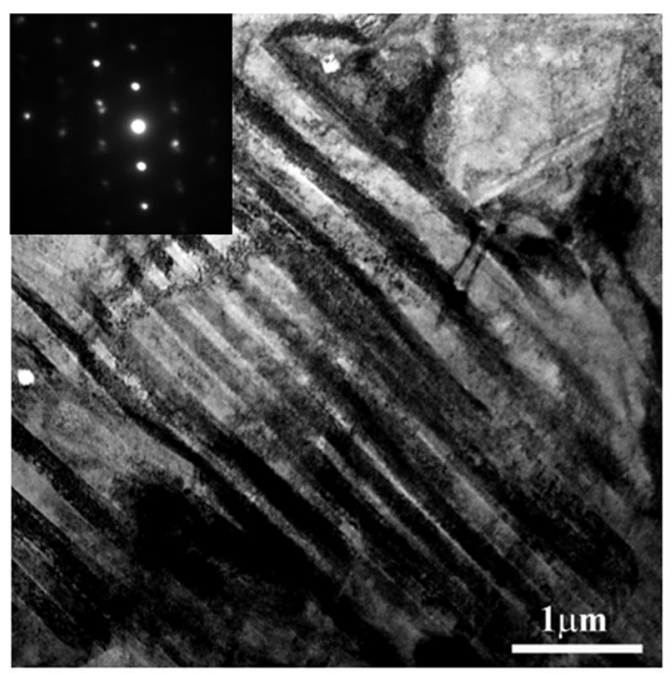

(a)

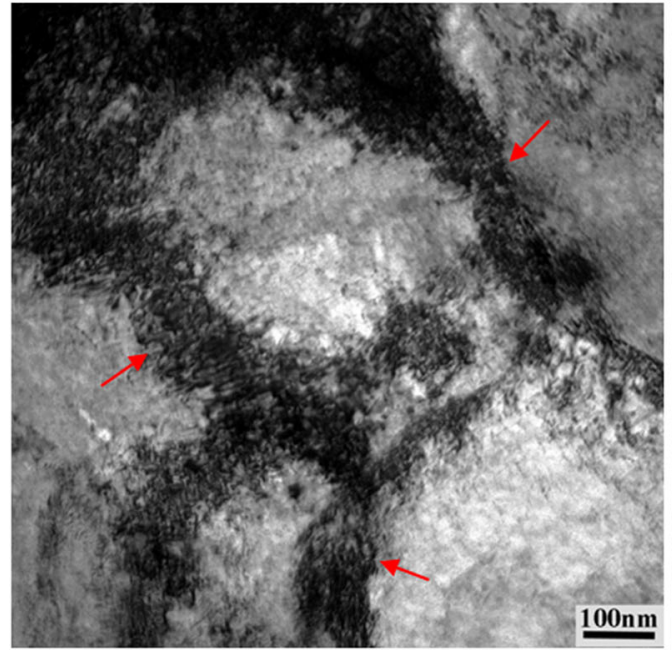

(c)

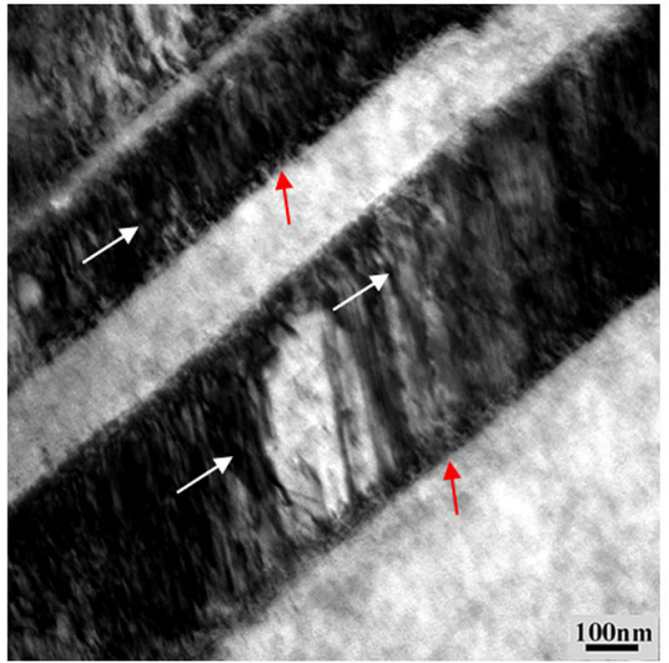

(b)

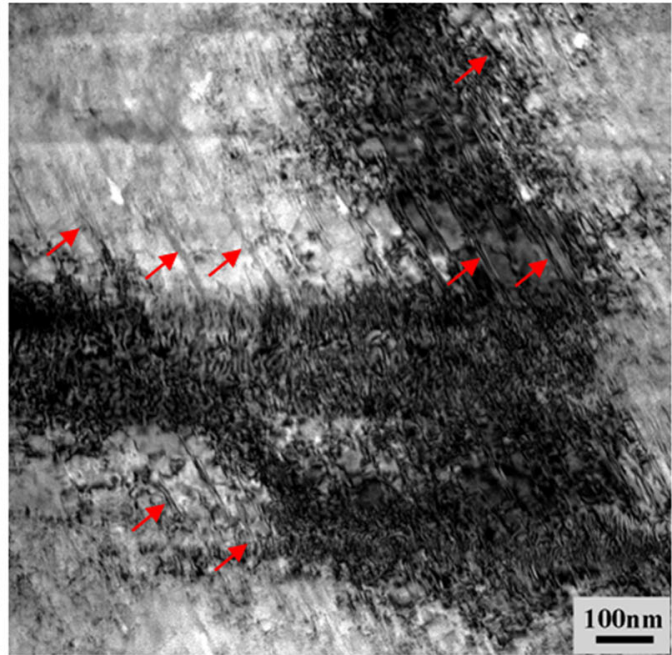

(d)

Fig. 13-Representative TEM micrographs of sample B8. (a) Formation of deformation twins, diffraction pattern from twins [110] secondary micro-twins; $(c)$ formation of cell-dislocation substructure; $(d)$ interaction of thin twins with the dislocations formed as a result of deformation.

\section{CONCLUSIONS}

A study of preferable deformation modes in a TWIP steel sheet using TEM examination and modeling simulation for different strain paths obtained by stretch forming samples of various shapes and tensile tests has been performed. This study has shown that the strain path influences the activated deformation mode and, therefore, formation of microstructure.

It was shown analytically that for a cube-oriented single crystal, in the absence of rotation with respect to the principal axis of the strain tensor, the strain path and the level of accumulated equivalent strain define the preferable deformation mode.
Numerical simulation results for polycrystalline material confirmed that the strain path influences the deformation mode and twin-to-slip ratio, especially at small levels of equivalent strain. Twin-to-slip ratio changes with strain path, which leads to different twin volume fractions for different locations of samples. The deformation by twin occurs heavily in the initial stage after which the contribution of twinning to deformation rapidly decreases.

The simulation has also shown that the twinning activity is the highest in tensile deformation, followed by the bi-axial and the plane strain cases, which are quite similar in twinning volume fraction. 


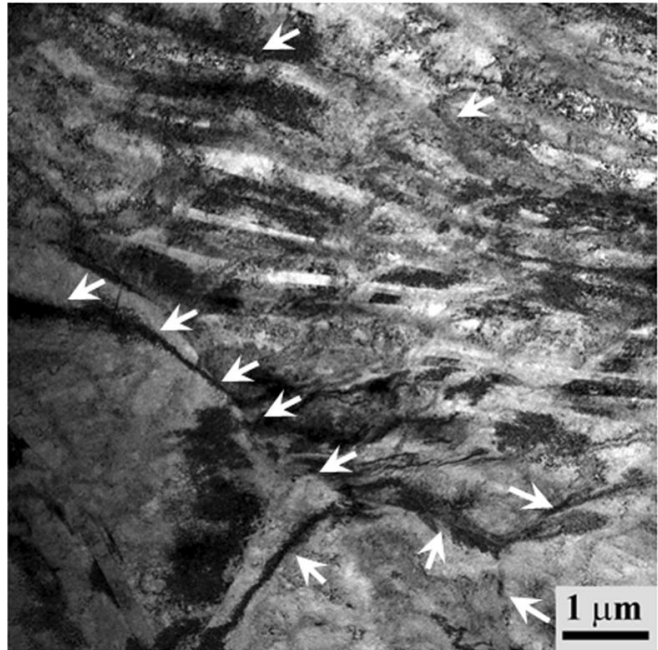

(a)

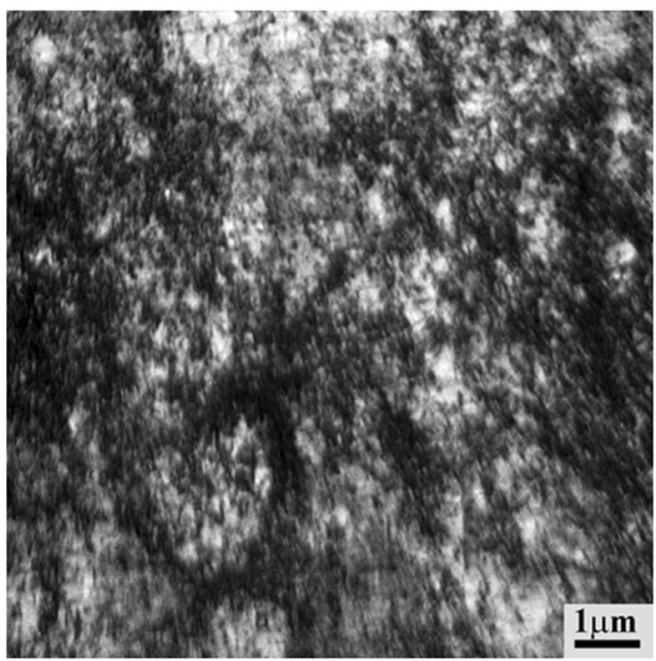

(c)

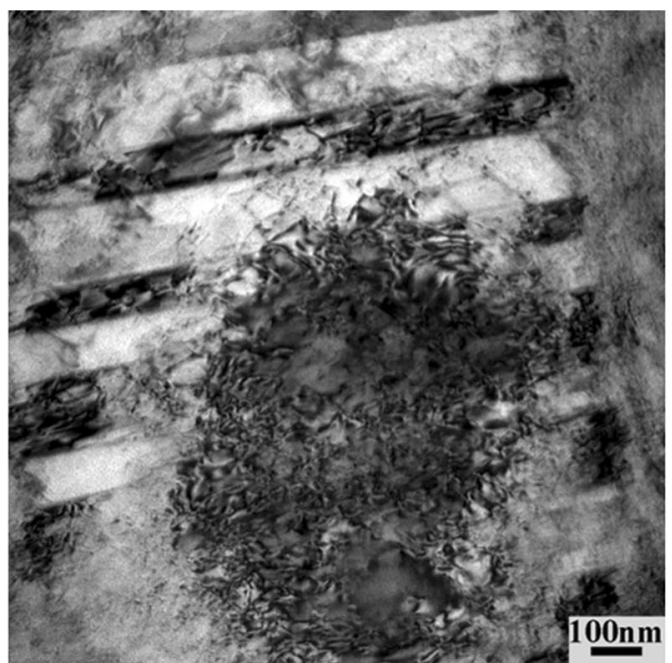

(b)

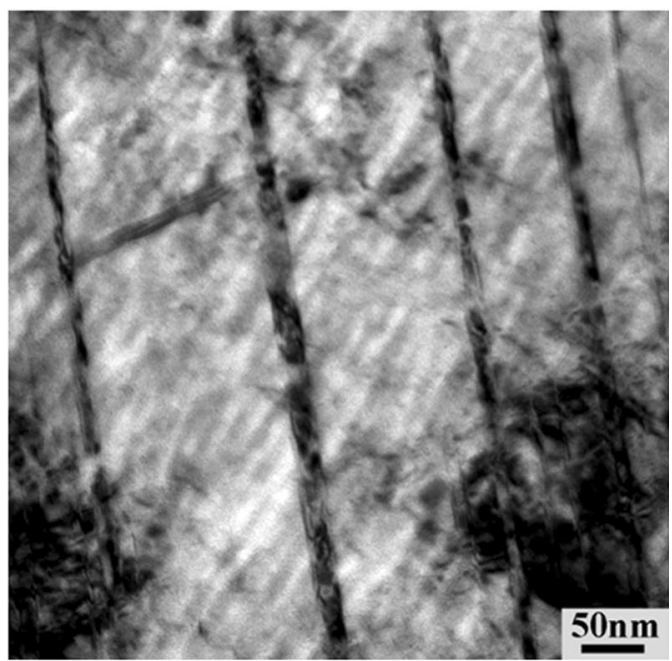

(d)

Fig. 14 - Representative TEM images from samples P2 and P3. (a) Formation of deformation bands (P2), arrows indicate the grain boundary; (b) interaction between the dislocations and deformation twins (P2); (c) cell-dislocation substructure (P3); (d) deformation twins (P3).

For the polycrystalline material with a large number of neighboring grains randomly oriented, the role of the strain path diminishes with increasing accumulated strain. TEM observations have confirmed the role of the strain path in activating different deformation modes and on the twin volume fraction. 


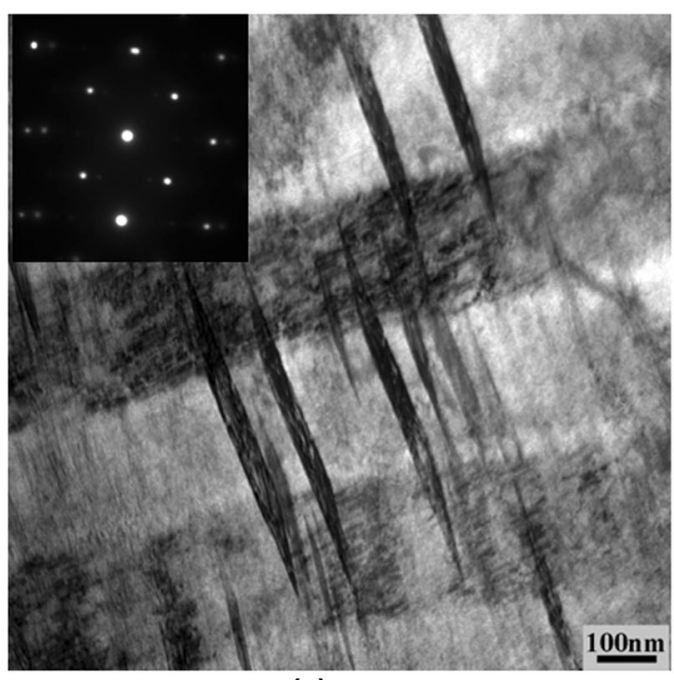

(a)

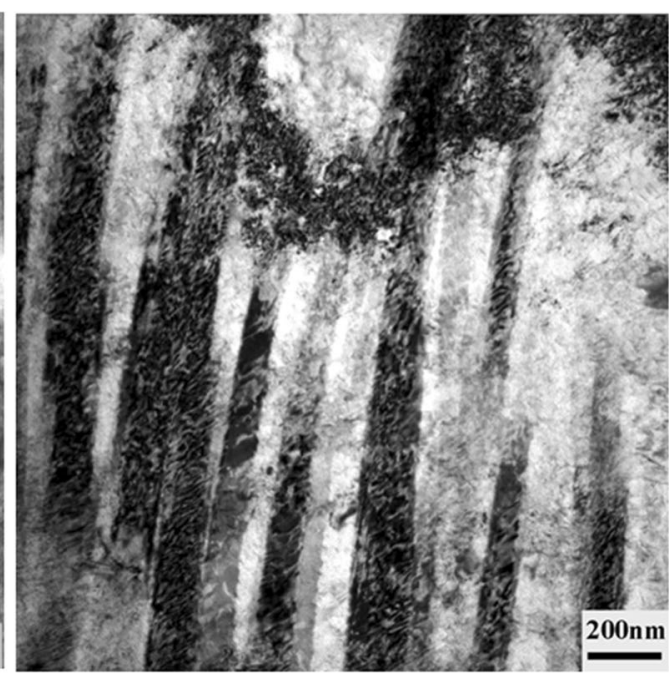

(b)

Fig. 15-Representative TEM images of samples. (a) P6; (b) P9.

\section{ACKNOWLEDGMENT}

The authors would like to express their gratitude to Professor Laszlo S. Toth from University of Lorraine (France) for fruitful discussion and access to the Metz-VPSC code for modeling of polycrystalline materials developed by him and his group.

\section{REFERENCES}

1. J.-K. Kim and B.C. De Cooman: Mater. Sci. Eng., A, 2016, vol. 676 , pp. 216-31.

2. O. Bouaziz, S. Allain, C. Scott, P. Cugy, and D. Barbier: Solid State Mater. Sci., 2011, vol. 15, pp. 141-68.

3. I. Karaman, H. Sehitoglu, K. Gall, and Y.I. Chumlyakov: Scripta Mater., 1998, vol. 38, pp. 1009-15.

4. I. Karaman, H. Sehitoglu, Y.I. Chumlyakov, H.J. Maier, and I.V. Kireeva: Scripta Mater., 2001, vol. 44, pp. 337-43.

5. W.F. Hosford: Mechanical Behavior of Materials, 2nd ed., Cambridge University Press, Cambridge, 2005, pp. 166-75.

6. J.W. Christian and S. Mahajant: Prog. Mater Sci., 1995, vol. 39, pp. 1-157.

7. K. Renard, H. Idrissi, D. Schryvers, and P.J. Jasques: Scripta Mater., 2012, vol. 66, pp. 966-71.

8. L. Bracke, L. Kestens, and J. Penning: Scripta Mater., 2009, vol. 61 , pp. $220-22$.
9. I. Gutierrez-Urrutia, S. Zaefferer, and D. Raabe: Mater. Sci. Eng., $A, 2010$, vol. 527, pp. 3552-60.

10. P. Yang, Q. Xie, L. Meng, H. Ding, and Z. Tang: Scripta Mater., 2006, vol. 55, pp. 629-31.

11. L. Meng, P. Yang, Q. Xie, H. Ding, and Z. Tang: Scripta Mater., 2007, vol. 56, pp. 931-34.

12. I. Gutierrez-Urrutia and D. Raabe: Acta Mater., 2011, vol. 59, pp. 6449-62.

13. Z. Chen, X. Zhang, C. Liu, Z. Zhou, and S. Li: J. Mater. Sci., 2002, vol. 37, pp. 2843-48.

14. U.F. Kocks, G.R. Canova, and J.J. Jonas: Acta Metall., 1983, vol. 31, pp. 1243-52.

15. Z. Chen, H. Cai, S. Li, X. Zhang, F. Wang, and C. Tan: Mater. Sci. Eng. A, 2007, vol. 464, pp. 101-09.

16. I.J. Beyerlein, L.S. Toth, and S. Suwas: Phil. Mag., 2007, vol. 87, pp. 885-906.

17. GOM mbH: http://www.gom.com.

18. C.F. Gu, M. Hoffman, L.S. Toth, and Y.D. Zhang: Mater. Charact., 2015, vol. 101, pp. 180-88.

19. C.F. Gu, L.S. Toth, and M. Hoffman: Acta Mater., 2014, vol. 62, pp. 212-24.

20. A.A. Saleh, C. Haase, E.V. Pereloma, D.A. Molodov, and A.A. Gazder: Acta Mater., 2014, vol. 70, pp. 259-71.

21. S.R. Kalidindi, C.A. Bronkhorst, and L. Anand: J. Mech. Phys. Solids, 1992, vol. 40, pp. 537-69.

22. V. Shterner, I. Timokhina, and H. Beladi: Mater. Sci. Eng. A, 2016, vol. 669, pp. 437-46.

23. J. He Ji and D. Zeng: X. Zhu. C. Xia, S. LiInt. J. Solids Struct., 2014, vol. 51, pp. 402-15. 